\title{
On Branched Chain Processes, the Laws of Development of Which Are Expressed by Numerical Sequences Like Fibonacci Numbers, a New Look at Their Nature
}

\author{
Yurii Petrenko \\ Medical and Biological Faculty, Pirogov Russian National Research Medical University, Moscow, Russian Federation
}

Email address:

yury_petrenko@mail.ru

To cite this article:

Yurii Petrenko. On Branched Chain Processes, the Laws of Development of Which Are Expressed by Numerical Sequences Like Fibonacci Numbers, a New Look at Their Nature. Science Journal of Chemistry. Vol. 6, No. 4, 2018, pp. 30-42. doi: 10.11648/j.sjc.20180604.11

Received: July 23, 2018; Accepted: August 15, 2018; Published: October 11, 2018

\begin{abstract}
Branched chain chemical reactions represent a special class of chemical transformation reactions of matter, for the discovery and experimental-theoretical development of which Semenov N. N. and Hinshelwood C. N. were awarded the Nobel Prize in 1956. In nature, such processes are widespread. Objective. To investigate the nature of various numerical sequences of the Fibonacci type and to find out under what conditions they can reflect (express) the patterns of development of branched chain processes. In this work, the state is formulated that branched chain chemical reactions are a particular case of branched chain processes of any nature in different spheres, including biological. It is shown that many branched chain processes can generate numerical sequences of Fibonacci, Lucas, Shannon and others of the same type, which reflect the dynamics of their development. For all the indicated numerical sequences, it is typical that their formation is determined by a general recurrent law, which has been the subject of research for many well-known mathematicians. For each of the indicated numerical sequences they established other laws alternative to the recurrent one, however, they were all of a private nature. They were in accordance with the recurrent law only in the case of one specific sequence. Comparative analysis of many numerical sequences allowed us to find a universal law that is common for all types of sequences and terminologically define it as the law of "doubling with subtraction." For all numerical series the formation of which follows a recurrent law, the law "doubling with subtraction" is equally valid. The opposite is not true since there are numerical sequences that obey only the law of "doubling with subtraction," and the recurrent law is not valid for them. It means that the new law is more fundamental and, in fact, is the primary law and the recurrent law is secondary. Significant differences also exist in the consequences of these two laws. For example, the increments of sequences formed according to the recurrent law and the law of "doubling with subtraction" have fundamental differences in their mathematical expression, although they lead in different ways to the same result, namely, to $\Phi$ $=1,618 \ldots$ with the serial numbers of the sequence terms tending to infinity. In the work for each of these laws, a branched chain biological process that was unique to it was found and put in correspondence. In the case of the law of "doubling with subtraction", the process was followed by the termination of chains with characteristic parameters: the chain length is three links, the branching factor is -2 . In the case of a recurrent law, the process was without chain termination, with an infinite length and with a branching factor of -2 , and with some delay limiting the branching. It seems interesting that such different branched chain processes of different character are described by the same Fibonacci sequence. The processes of branched chain character corresponding to the sequences of Lucas, Shannon, and others are discussed. Conclusions. According to our work, it follows that all formal mathematics, that is, all the mathematical features and patterns related to the Fibonacci sequence is just a description of those features and patterns that are inherent in the branched chain processes that actually produce these and other sequences.
\end{abstract}

Keywords: Branched Chain Processes, Numerical Sequences, Fibonacci Sequences, Lucas Numbers, Recurrent Formula 


\section{The Usage of the Numerical Fibonacci Sequence in Biology and Medicine}

Fibonacci (Leonardo of Pisa), well-known Italian mathematician, about whom, G. Polya, a prominent modern mathematician and educator, said: "It was an outstanding Italian mathematician, perhaps the most brilliant scientist of all mathematicians of the whole European Middle Ages [1]. Among the mathematical problems that Fibonacci (FB) solved, there was one about many pairs of rabbits might be born from a single pair at certain conditions and he (FB) gave a solution to this problem in the form of a numerical sequence: $1,1,2,3,5,8,13,21$,..etc. This numerical sequence was later called the Fibonacci numbers or Fibonacci sequence or even simply - FB. G. Polya himself used a Fibonacci sequence in his research. Besides, he paid special attention to the recurrent law of its formation, expressed in the form: Fn $=$ Fn- $1+F n-2, n=3,4,5 \ldots$, and initial conditions $F 1=1, F 2=1$. Polya, as mathematician, was more interested in mathematical side of this recurrent law. The increment of this numerical FB sequence for $\mathrm{n}$, aspiring to infinity, has the value $\mathrm{Fn} / \mathrm{Fn}-1 \approx 1,618 \ldots$ That is why there are reasons to identify the concept of the FB number and the "golden ratio". The golden ration is a rule about how parts and integer must be related to each other in order to be in harmony and for some reason this harmony is expressed by an irrational number $1,618 \ldots$ In biological and medical literature a lot of attention is given to a question about the usage of Fibonacci sequence, which increment is connected with the golden ratio. According to data of this literature, in recent years, the work on the application of the Fibonacci sequence as a methodological basis for the first stage of clinical trials of various drugs, mainly anticarcinogenic agents, began to appear in large quantities. In work [1] it is stated that the FB sequence and its modified version are the most used methodologies for determining the dose increments which are used in the first phase of clinical drug trials. In the same work, an interesting explanation is given about why the FB sequence is widely used. The main thing is that this is one of the first methods described for these purposes. It is easy to understand for patients, investors and regulators (such as the Ethics Committee). Some authors believe that the FB sequence is preferable when the dosetoxicity curve has a sharp steepness [2, 3], according to animal toxicology. It is claimed that about $50 \%$ of the current clinical trials of the first phase are still conducted using FB sequence, and at the same time, the modified FB sequence is currently the most used [2]. It should be noted that the first phase of clinical trials of anticarcinogenic agents is the first development of human trials and it is an important step in drugs creation. On this first stage (dose-orientational), clinical trials are conducted to determine the optimal recommended dose for a new compound for further testing in the second phase of the trials [2]. For cytotoxic drugs, this dose corresponds to the highest dose associated with an acceptable level of toxicity which is related to the maximum tolerated dose. From the point of view of efficiency, it is clear that it is important to move up to the maximum tolerated dose, and it has to be done quick, but as long as it is safe, but it is unknown where the safe point is. Therefore, there is a problem about minimizing probability of getting too low or too high doses for patients. In the literature it is noted that the contradiction between safety and efficiency in the first phase of clinical trials will be solved with the help of new methods, which are in development. In most oncological trials of the first phase, it is often claimed, that the dose increment was corresponded to a modified FB sequence. At the same time, there is some dissatisfaction in the literature about the dose escalation and its defining laws. It is noted that even the term in the method of the modified FB sequence is vague and incomprehensible. There is no clarity about the nature of the FB sequence itself. Further, the questions will be considered in detail both about their nature and what they reflect. There should be the law of dose escalation by the type of a numerical sequence of FB or another type, at least for standardizing the conditions for testing the first phase. We believe that our work will give a greater understanding of their nature and semantic content. For example, let us mention several recent works about the application of the numerical FB sequence in clinical trials of the first phase. In 2015, a large group of American researchers from various cancer research facilities in Texas (12 authors) published a work about the first phase of clinical trials of the Bi-shRNA STMN1 BIV drug against hard-to-treat cancer type [4]. In the same year, a large group of South Korean authors published an article of the first clinical trials about determination the pharmacokinetic characteristics of DHP107 in humans, a new oral drug composed of lipid ingredients and paclitaxel [5]. The preparation DHP107 demonstrated efficiency, safety and pharmacokinetics of intravenous application, comparable with paclitaxel as the second line of therapy in patients with advanced gastric cancer. The dose escalation law with the modified FB sequence was used In both of these studies. Quite a lot of publications of this kind about the use of FB numbers to determine the necessary doses and medicines is contained in the literature of recent years. In recent years, authors have begun to focus on more fundamental things, the important role of Fibonacci numbers and associated with them golden ratio in the biological and medical spheres. Noteworthy is the work of Japanese authors: "Two Golden Ratio indices in fragment-based drug discovery" [6]. Fragment-based drug discovery (FBDD) is a new scientific direction that can be translated as a fragmented basis for drug development. It is identified with low molecular ligands $(\sim$ $150 \mathrm{Da})$, from which highly reactive molecules with theoretically drug-like properties are collected. It was the product of the latest scientific achievements and, in the opinion of some researchers, this trend seems promising [7], and the fact that the Fibonacci numerical sequence is involved in it causes a great interest. In this work, two new indexes based on the "Golden ratio" are proposed, which increase the effectiveness of drug development based on the 
FBDD method. The authors of this work method selected thirty examples from a large number of literature sources about the use of the FBDD, in which its application was successful, and analyzed it. They analyzed the ratio of the number of heavy atoms (not hydrogen) entering the lead drug to the number of heavy atoms that make up the original fragment (Scaffold part), and the same ratio of the number of heavy atoms between the scaffold part and evolution part. From the graphs of distributions of frequency of occurrence, the obtained values of these ratios, based on the data of thirty used examples, they saw a sharp peak of the maximum. It corresponded to the values of these ratios in the region of 1.618 for both cases. These results were then reinforced by the thermodynamic data of both the theoretical and experimental plan obtained by the authors. In the same study, the authors discovered yet another circumstance related to the Fibonacci numbers. They found that the percentage of ligand efficiency, which is an important parameter used in FBDD, is determined by the value of $F$. Based on the obtained data, two indexes were proposed, which make the FBDD process more specific and increases its efficiency. Interestingly, based on an analysis of the merits and capabilities of this method, the study states that it creates a new era in the drug development. Another interesting point of the work is that the authors are wondering why the $\mathrm{Fb}$ numbers and the golden ratio appear in the FBDD method and they hope that this understanding will be achieved in the future. Another important example of the fundamental connection between biological and medical phenomena and processes with $\mathrm{Fb}$ numbers can be found in this study [8]. In this study, the authors analyze both theoretically and experimentally the relationship of two drugs used in the form of their combination in inhibiting the channels. Such combinations are often used in clinical practice to investigate the mechanisms of action of drugs. For example, Schild-analyzes, which uses agonists and antagonists, can open a mechanism of competitive action. The authors of this work considered two different types of channels that are blocked by two substances due to their reversible binding to sites located inside the pore. These channels are terminologically defined in quantitative pharmacology as Syntopic and Allotopic Models [8]. Syntopic Model included one binding site for both drugs, and Allotopic Model is two different sites. It was believed that the binding of any single substance is already accompanied by the closure of the pore. Channels remain open if their connecting sites are free. After a rigorous analysis of the inhibition of these two types of canals by each of the substances separately and with their joint participation, differences were determined between them by this criterion. As a result, a cubic equation $\mathrm{y} 3-2 \mathrm{y}+1=0$ was obtained, which solution, under certain conditions, surprisingly led to a golden ratio and interesting consequences (giving the roots 0.618. and -1.618..). This circumstance is also surprising because, as will be shown below, a similar cubic equation resulting three roots, two of which refer to the golden ratio, was obtained by analyzing the law of doubling with subtraction which was discovered by us. These are two examples from all literature, when the solutions of the golden ratio obtained from the cubic equations describing completely different processes by their nature. The authors of this work attempted to verify their results experimentally at the receptors 5-hydroxytryptamine type 3 (5HT3) using known channel blockers. Unfortunately, there is no clear data on this in the study. Another very interesting work is the work on the connection of Fibonacci numbers with nucleotide frequencies in the human genome [9]. In this study, a mathematical model is presented which, based on two quite convincing assumptions, can accurately predict nucleotide frequencies in single-stranded human DNA using Fibonacci numbers. The idea of the mathematical model was based on the analogy with the Fibonacci numbers. In the relationship between two neighboring terms of the continuously increasing numerical FB sequence, there always exists a limit which is numerically equal to F-value. The main assumption accepted in the construction of this mathematical model, that this is similar to nucleotide frequencies with the growth of the number of nucleotides. There are still some assumptions, but they are less important than the one mentioned above. This mathematical model was based on the numerical $\mathrm{Fb}$ sequence and the recurrent law of its formation. The second rule of Chargoff's correspondence and several more plausible assumptions were also used. It is known that the first rule of Chargoff's correspondence is related to double-stranded DNA, and was used by Watson and Crick to substantiate their double-stranded DNA model. The second rule of Chargoff's correspondence relates to single-stranded DNA. For this case, a mathematical model has been developed. The data obtained from this mathematical model, applied to all 24 human chromosomes, produces surprisingly accurate results of nucleotide frequencies for all nucleotides. Unfortunately, the developed mathematical model seems rather abstract, and it is difficult to reveal why it has such predictive power. Apparently, the comprehension of this with the link between the meaning and the numerical series of the FB is still ahead. Nevertheless, the developed mathematical model makes it possible to raise the level of understanding of the phenomenon contained in the second rule of Chargoff's correspondence in addition to the explanations found in [10]. Another important scientific direction, which is widely represented in numerous publications, concerns examples of the detection of manifestations of regularities of the FB and the golden ratio in various medical phenomena and processes. In this case, the publication [11] deserves attention, which presents the first study of the connection of the Fibonacci cascade to the distribution of coronary artery damage in the human heart responsible for elevation of the ST segment in myocardial infarction. In this study, the appearance of $\mathrm{Fb}$ numbers in distribution of the damage of the coronary arteries in the human heart is demonstrated. The authors of this study believe that the predisposition to this relation appears in nature, perhaps because this ratio optimizes the packing efficiency of structures in a confined space in such a way that unused space is minimized and the supply of energy or 
nutrients is optimized [11]. In the anatomical structure of living organisms and their parts, we often find confirmation in the regularities peculiar to the numerical $\mathrm{Fb}$ sequence. In particular, recently appeared an increased interest in the anatomical structure of the human body and its parts, mainly, the hands. It is connected, apparently, with the development of a perfect functional robotic arm, the creation of which is being fought by many researchers. They need knowledge of the regularity of the anatomical structure of the hand and its functional characteristics. In this case, we can note the work [12], where from 100 healthy male volunteers of Chinese origin, data of the functional proportions of the arm approximated by the numerical Fibonacci series is presented. A statistical analysis of the data was done and results were presented in confirmation of their connection with the numerical data of the FB sequence. In the American journal of otolaryngology, there is an article about the regularities in the structure of the cochlea and other spiral forms, which common in nature and arts and their relationship to the numerical sequence of $\mathrm{Fb}$ [13]. The law of the golden ratio is used in medical oncological practice as a method for establishing the exact location of the navel in women after surgical operations [14]. The meaning of this need is aesthetic. In this study, it is believed that this method favorably differs from others used for these purposes. Many other medical are also discussed in the literature. For example, is there a relationship between systolic and diastolic blood pressures and the golden ratio [15]. In the questions of psychology, a golden ratio also appears. At the same time, there are works whose authors directly express their dissatisfaction with the fact that the widespread use of $\mathrm{Fb}$ numbers in the first phase of clinical trials is done under conditions when their very nature is not clearly known and, especially, this applies to the modified Fb numbers. The data presented on the use of the FB numerical sequence show how significant this problem is for medicine and biology. At the same time, the questions about the nature of the Fibonacci numbers and the nature of their connection with the phenomena and processes observed in the medical and biological spheres remain open. In a number of works the opinion is expressed that they can be solved in the future. Objective: Based on the new law, which is more fundamental than the alternative recurrent law for the formation of $\mathrm{Fb}$ numbers, reveal the nature of the phenomena and processes that cause their appearance and which they describe as processes that have a branched chain nature.

\section{About Branched Chain Reactions}

In 1956, the Nobel Prize for scientific development of the problem of branched chain chemical reactions was awarded to the Nobel Committee. One of the laureates of the award was Semenov N. N., whom we consider to be the founder of chemical physics, the creator of the quantitative theory of chain reactions. The second winner Cyril Norman Hinshelwood was awarded the Nobel Prize, according to the encyclopaedic interpretation, for the theory, kinetics and mechanisms of chain branched reactions. Academician N. N. Semenov in his Nobel speech, read in Stockholm, in connection with the awarding of the Nobel Prize in 1956, which was devoted to the discovery of a vast class of chain chemical reactions, determined and characterized branched chain reactions. This speech was published in our book as a separate brochure and in the journal «Advances of Chemistry» [16]. At the end of the Nobel speech, N. N. Semenov noted that many chemical reactions, which we know as simple, can be chain or branched chain when examined deeper. The "active particle" is the fundamental concept of the theory of branched chain chemical reactions. This concept was introduced into scientific circulation by $\mathrm{N}$. $\mathrm{N}$. Semenov and became an encyclopedic concept. In a chain reaction, the primary active particles appear, causing a long series of successive transformations of the substance. These reactions of continuation or development of the chain are possible because in the interaction of the active particle with the initial substance, in addition to the final substance, an active particle is formed which also reacts with the starting material, etc., until for some reason a "death " of this particle happens which is a chain break. The active particle, which cyclically does something with the medium, while not changing itself, will be an example of a simple chainunbranched process. In particular, such an example of an active particle can be the enzyme molecule in a simple enzymatic process or any other catalyst in the catalytic process. Other leading concepts and parameters in the theory of branched chain processes that occur under certain conditions that determine their nature and mechanism of action are the chain length and the branching factor. The length of the chain is the number of its links from the termination to the other termination, meaning how many times during this interval the active particle reacted. Coefficient of branching is the number of active particles appearing after each act of continuation of the chain. If the branching ratio is 1 , then this is just a chain process. In the case when two or more active particles appear after the interaction of one active particle, the branching factor is 2 or more and this is a branched chain process. According to the ideas of N. N. Semenov: "Such reactions are the most common type of chemical transformation of substances." This direction of branched chain reactions continued to develop successfully and found expression in the reactions of valuable lipid peroxidation, having important role to biology and medicine. The role of an active particle is played by various radicals, in particular peroxide nature. It should be noted that we also made some contribution to the development of this problem, both theoretically and experimentally. In particular, in solving the problems of inhibiting the free radical chain process of lipid peroxidation by antioxidants, acting both by the chain termination mechanism and by the mechanism of inhibition of their branching, having a list of published works in this direction, a link of one of them [17]. It should be noted that even now a lot of studies are published, in particular on medical topics, in which membrane peroxide oxidation of lipids is studied, also 
its role and importance for the functional state of cells, organs and tissues and the organism as a whole. Branched chain processes that occur in the organism are not limited to this. For example, has been discovered the phenomenon of branched chain oxidation of oxyhemoglobin under the influence of certain factors, hydrogen peroxide with complete inhibition of catalase or nitrite ions [18]. Everything considered above was from the category of branched chain reactions of chemical nature, the direct chemical transformation of substances. However, in addition to the chemical reactions associated with the transformation of substances, there are classes of other phenomena that also proceed as branched chain mechanism, which is a whole layer of processes not directly related to chemistry, which were considered as phenomena and processes of the other nature. It can be assumed that the concepts and terminology developed for branched chain chemical reactions should be fair and applicable to them too. The implementation of such non-chemical processes is inevitably associated with the existence of active particles in them. Using the terminology of the Nobel laureate N. N. Semenov, a pair of rabbits can formally be regarded as active particles. A pair of rabbits, like an active particle, leads the chain, continues it and generates new chains, which means that it makes a branching of the chains. This process of multiplying and increasing the number of pairs of rabbits will occur through a branched chain mechanism.

Below we will consider the regularities and characteristics of such processes and show that they are responsible for the appearance of a numerical sequence of FB and other numerical series.

\section{The Numerical Sequences of Fibonacci, Lucas and Shannon, Their Characteristics and Laws That They Are Determined by}

Earlier we showed FB sequence, which can be defined as canonical and also noted that this was not its only form. It is also represented as a series of $1,2,3,5,8,13$, etc. and a sequence of the mathematical form $0,1,1,2,3,5$, 8, etc. For both of them, a recurrent law is valid, stating that each following term, starting with the third, is equal to the sum of the two previous ones. G. D. Cassini discovered a new law for the formation of a numerical Fibonacci sequence that is alternative to a recurrence law, which connects three neighboring numbers in FB sequence of the following form: $\mathrm{F}^{2}(\mathrm{n})-\mathrm{F}(\mathrm{n}-1)^{*} \mathrm{~F}(\mathrm{n}+1)=(-1)^{\mathrm{n}+1}$. After experimenting with this equation, one can make sure that it is directly valid only for the canonical form of the FB series and it is individual. In the 19th century, the French mathematician Lucas discovered yet another law for the formation of the FB series, and, in two of its expressions, taking into account the evenness of the serial number of its terms. This law is expressed by the following expression: $\mathrm{F}_{2 \mathrm{n}}=\mathrm{F}^{2}{ }_{\mathrm{n}+1}-\mathrm{F}_{\mathrm{n}-1}^{2} ; \mathrm{F}_{2 \mathrm{n}+1}=\mathrm{F}_{\mathrm{n}+1}^{2}+\mathrm{F}_{\mathrm{n}}^{2}$

The experimental verification, which is easy to perform by substituting numerical values into these expression, confirms their fidelity, however, directly only for FB of canonical forms. And further on, the mathematical formalism applied to this sequence led to the establishment of a whole series of identities and the regularities inherent in it. One of them, discovered by the French mathematician Binet, takes a special place among them. The peculiarity is explained by the fact that the law of formation of FB, is radically different from all others, because it determines the terms of the sequence by the golden number F, equal to 1.618 .., obtained from the solution of the golden ratio problem. It should be noted that back in 1595, Johannes Kepler, an outstanding astronomer and mathematician, noticed one important peculiarity of the numerical FB sequence. He noticed that the ratio of the next term to the previous one while the sequence increases in the direction towards infinity tends to $1.618 \ldots$ Which means, a connection between the numerical FB sequence and the golden ratio has been established.

We see what outstanding people, mentioned by us, were dealing with this problem. Also, by works of other, less prominent researchers many more laws have been established which characterize and determine the properties of the FB sequence. The French mathematician Lucas created a new series of numbers, completely different, but the recurrent law of their formation was the same as for the FB sequence. This numerical series has the form: $1 ; 3 ; 4 ; 7 ; 11 ; 18 ; 29 ; 47 ; 76$; $123 ; \ldots$ etc. The first two terms are also the initial conditions, as for a FB. This sequence is called Lucas sequence, and its elements - Lucas numbers. An important feature of Lucas sequence is the particular law of its formation, discovered by the mathematician Binet, by using golden number $\Phi$ having the simplest form: $F_{n}=\Phi^{n}-\Phi^{-n}$ и $F_{n}=\Phi^{n}+\Phi^{-n}$, respectively for odd and for even "n". He also established another law for the numerical FB sequence, but it has difficult (complex) form. If the numerical sequences of FB and Lucas has the same recurrent law, and the sequences are cardinally different from each other, then it means that this is due to its different initial conditions.

The initial conditions $(1,1)$ and $(1,2)$ give the same FB sequence. The initial conditions (1.3) give us another numerical sequence, called the Lucas sequence. Taking the initial conditions (1.4), we get the third kind of a numerical sequence formed according to a recurrence law, with the form: $1,4,5,9,14,23,37 \ldots$ etc. Choosing other initial conditions (1.5), we get the following numerical sequence: 1 , $5,6,11,17,28,45 \ldots$ etc. The last two sequences can be called Shannon sequence, since he was the first who drew attention to them and published them [19]. The verification of the applicability of the Cassini, Lucas and all other known laws to the Lucas sequence and two Shannon sequences gives a negative result. This means that all these laws are specific only for canonical form of FB sequence. On the other hand, all known laws that establish connections between numbers of Lucas sequence are not applicable to either the FB sequence or the Shannon sequence. This means that all these laws are specific only for one specific sequence. And now we can discuss a new law which was discovered by us after studying these sequences. As a result, we established 
new law which is radically different from all previously known laws. The formulation of this law: "Any term in the FB sequence and its value is determined by the doubled value of the previous term minus the value of the third previous term". Initial conditions for it will be the first three terms of the sequence. If this law is expressed analytically, then it has the following formula: $\mathrm{F}(\mathrm{n})=2 * \mathrm{~F}(\mathrm{n}-1)-\mathrm{F}(\mathrm{n}-3)$, where $\mathrm{n}=$ $3,4,5, \ldots$, etc - is any determinable sequence number of $\mathrm{FB}$, and $F(n)$ is its value, $n-1$ its previous term with the value $F$ (n-1) and n-3 the third number with the value F (n-3). It is not difficult to verify the truth of the relation between different terms of any of the sequences presented here (FB, Lucas, and two Shannon sequences). Thus, the resulting relation is the law determining the formation of the numerical sequence of FB, Lucas, two Shannon sequences, and also many other numerical sequences. In the literature, this law does not occur and is not mentioned, which allows us to call it new and terminologically define as the law of "doubling with subtraction" (DWS). In this terminology, it reflects its greater fundamentality and generality in relation to all numerical sequences. Let us remind that in literature the recurrent law is considered to be the main, fundamental and it is the basis of definition of the numerical FB sequence.

\subsection{Features Exhibited by Numerical Sequences in Their Formation According to a Recurrent Law and According to the Law of "Doubling with Subtraction"}

\subsubsection{About the Similarities and Differences Between the Law of "Doubling with Subtraction" and Recurrent law for the Fibonacci Sequence}

The fact that these laws appear to be identical with each other follows a direct verification of the results obtained by using them. They are obtained exactly the same for all the

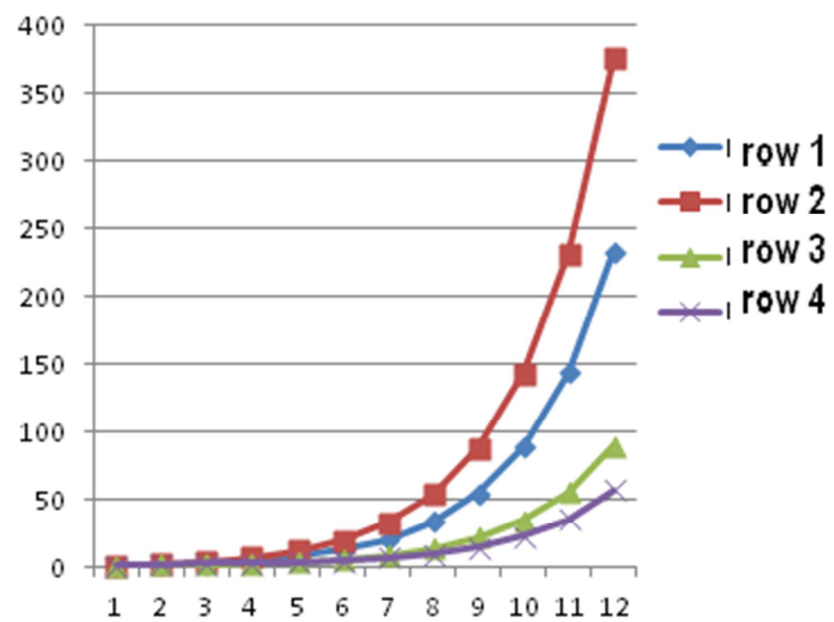

sequences listed above. This apparent identity is confirmed theoretically. Indeed, the mathematical expressions of these laws have the form: $\mathrm{F}(\mathrm{n})=\mathrm{F}(\mathrm{n}-1)+\mathrm{F}(\mathrm{n}-2)$ and $\mathrm{F}(\mathrm{n})=2 * \mathrm{~F}$ (n-1) - F (n-3). Combining them, subtracting the second equation from the first one, we obtain $\mathrm{F}(\mathrm{n}-1)=\mathrm{F}(\mathrm{n}-2)+\mathrm{F}$ (n-3), the same recurrent law. The main goal of this article is not about showing new law, our goal more significant. We reasonably assert that the law discovered by us is the primary and fundamental law for the formation of Fibonacci numbers in its numerical sequence. A well-known recurrent law, which is listed everywhere as the main and determining one, is not main at all. It is secondary to the law that we discovered, it is its consequence, and we will justify it below. The fact that the recurrent and our laws are valid for all sequences means that they are universal, all other laws are particular. Therefore, we have two universal laws. Which one of them is the main, and which one is secondary, can be answered this way: We take the canonical FB sequence and arbitrarily add to each of its terms any same number, let it be number " 1 " for simplicity. As a result, we get sequence: 2,2 , $3,4,6,9,14,22$, etc., which does not obey the recurrent law and at the same time the law that we discovered works absolutely fine. It works the same way if we subtract this number " 1 " from sequence numbers instead of adding it. After subtracting, we get the sequence: $0,0,1,2,4,7,12$, 20 ...etc. If we remove the first two terms " 0 " we can see that it still does not obey the recurrence law, but obeys the law discovered by us. The DWS law is also valid for other sequences formed according to the formulas shown in Figure 1. These sequences were obtained by a different modification of the recurrent law, therefore, these are not sequences of Fibonacci type, since they no longer obey the recurrence law. At the same time the DWS law remains valid for them.

\begin{tabular}{|l|l|l|l|}
\hline 1 & 1 & 1 & 1 \\
\hline 2 & 2 & 2 & 2 \\
\hline 3 & 4 & 2 & 3 \\
\hline 5 & 7 & 3 & 3 \\
\hline 13 & 12 & 4 & 4 \\
\hline 21 & 20 & 6 & 5 \\
\hline 34 & 33 & 9 & 7 \\
\hline 55 & 54 & 14 & 10 \\
\hline 89 & 88 & 22 & 15 \\
\hline 144 & 143 & 35 & 23 \\
\hline 233 & 232 & 56 & 36 \\
\hline & 376 & 90 & 57 \\
\hline
\end{tabular}

Figure 1. Graphical view of Fibonacci sequence and some derivative forms.

On the abscissa axis - the serial numbers of the sequence, along the ordinate - their values. Sequences are shown in the columns on the right. Sequence 1 (row 1) - FB sequence which is formed by the formula $\mathrm{F}(\mathrm{n})=\mathrm{F}(\mathrm{n}-1)+\mathrm{F}(\mathrm{n}-2)$; sequence 2 (row 2 ), formed by the formula $F(n)=F(n-1)+$ $\mathrm{F}(\mathrm{n}-2)+1$; sequence 3 (row 3 ), formed by the formula: $\mathrm{F}$ (n)
$=\mathrm{F}(\mathrm{n}-1)+\mathrm{F}(\mathrm{n}-2)-1$; sequence 4 (row 4$)$, formed by the formula $F(n)=F(n-1)+F(n-2)-2$.

The last fact gives a reason to assert that the law discovered by us has a general nature, while the recurrent law is secondary to it and is valid only for more specific sequences. Our next step is a comparative analysis between 
the recurrent and the new law according to their differences and some characteristic indexes.

\subsubsection{About Specific Sequences of FB Type and Their Properties}

A large number of numerical sequences which formation follows the main recurrent law, which formula has the form: $F_{n}=F_{n-1}+F_{n-2}$, can be attributed to a certain type of sequences, determined as FB sequences, along with FB sequence itself. The question comes up: is there any numerical sequence of FB type, which simultaneously belongs to another type of numerical sequences, for example, geometric or arithmetic progression. This possibility exists only for a geometric progression. For the existence of such sequence, it should satisfy the conditions expressed by the following relations.

$$
\begin{gathered}
\quad F_{n}=a_{n}=a q^{n-1}, F_{n-1}=a_{n-1},= \\
F_{n}=F_{n-1}+F_{n-2}, a_{n}=a_{n-1}+a_{n-2} \\
a q^{n-1}=a q^{n-2}+a q^{n-3}, \\
q^{n-1}=q^{n-2}+q^{n-3},
\end{gathered}
$$

$F_{n}, F_{n-1}, F_{n-2} u a_{n}, a_{n-1}, a_{n-2}-$ terms of the FB sequence and the geometric progression with the indexes denoting their serial numbers, "a" is the first term, and "q" is the denominator of the geometric progression. The last equation is easily transformed into a quadratic equation of the following form:

$$
q^{2}-q^{1}-1=0,
$$

The resulting equation is identical to the known equation of the golden raiot and its solution will be the following two roots, q1 and q2.

$$
q_{1}=\frac{1+\sqrt{1+4}}{2} \approx 1,618 . ., q_{2}=\frac{1-\sqrt{1+4}}{2} \approx-0,618 .
$$

First root, the irrational number $1.618 \ldots$, in the special literature is usually denoted by the letter $\Phi$ [6]. Other root, the irrational number is $0.618 \ldots$ (with a minus sign), which is denoted by the letter $\varphi$ (the opposite notation also exists). The relationship between these numbers is determined by the relation $\Phi^{*} \varphi=1$. The presence of two roots means that in nature there are two numerical sequences that are formed according to a recurrence law, which means, they belong to the FB type, and which simultaneously are a geometric progression. The view of these two rows:

$$
\begin{gathered}
1, \Phi^{1}, \Phi^{2}, \Phi^{3}, \Phi^{4}, \Phi^{5}, \Phi^{6}, \ldots \text { etc. } \\
1,-\Phi^{1}, \phi^{2},-\phi^{3}, \Phi^{4},-\phi^{5}, \phi^{6}, \ldots \text { etc. }
\end{gathered}
$$

\subsubsection{About Special Sequences of the Class Formed by the DWS Law}

Consider the case where the FB sequence is formed according to the DWS law, which formula expression is represented in the form $F_{n}=2 F_{n-1}-F_{n-3}$, where $F_{n}, F_{n-1}$ and $F_{n-3}$ terms of the numerical Fibonacci sequence with their ordinal numbers, respectively, n, n-1 and $n-3(n>3)$. The question about the possibility of the existence of a numerical sequence obtained using DWS law and at the same time being a geometric progression is solved in a similar way, which means that the following equation must be: $a q^{n}=2 a q^{n-1}-a q^{n-3}$, where $a$ is the first term of the geometric progression, and $\mathrm{q}$ is its denominator. It is clear

that the value found for $\mathrm{q}$ will be a criterion for the possible identity between the geometric progression and the numerical sequence, meaning that the same sequences will be both a geometric progression and a numerical sequence formed by the DWS law. The equation $q^{n}=2 q^{n-1}-q^{n-3}$ can be easily transformed to $\mathrm{q}^{3}-2 \mathrm{q}^{2}+1=0$.

The resulting cubic equation can be solved by the Cardano method, in which, by appropriate substitution, it is reduced to an incomplete cubic equation for which the solution is sought and then the solution of the initial equation is sought using a solution of incomplete cubic equation. In our case everything is simple, since this cubic equation can be reduced to the form:

$\left(q^{2}-q^{1}-1\right)\left(q^{1}-1\right)=0$. Three roots are easily found using this cubic equation, its: $q_{1} \approx 1,618, q_{2} \approx$ $-0,618, q_{3}=1$. All of them are valid and two of them refer to the golden ratio. This is an amazing circumstance for us, since a similar cubic equation with roots, two of which also refer to the golden ratio, was obtained in one of the studies discussed above about the analysis of literature data of channel inhibition [8].

This can be verified by comparing the cubic equation obtained by us уравнение $\mathrm{q}^{3}-2 \mathrm{q}^{2}+1=0$ and other one $y^{3}-2 y+1=0$ which was obtained by authors of the study we mentioned before. Surprisingly, the analyzed phenomena, while being of fundamentally different nature, lead to a single outcome - the golden ratio. At the same time, our cubic equation is still somewhat different from the cubic equation obtained in the cited study. The second term of our equation has an exponent of 2 but in their equation - 1 . The results are also different. The first root is 0.618 , we have 1,618 , the second one is -1.618 , and we have -0.618 , sort of antisymmetric results. Why this happened and what kind of antisymmetric processes lie at their base deserves special attention. Apparently, there is something in common between the processes of inhibiting the channels and all the numerical sequences defined by the DWS law.

This may be caused by a branched chain mechanism, which is responsible for the observed phenomena. Note that from the point of the chain processes that we are developing here, many things, in particular enzymes, can be considered as an active particle. It also should be noted that the presence of three roots in our case means that there are three numerical sequences in nature that are formed by DWS law and which are a geometric progression at the same time. The first sequence is infinitely increasing, the second is infinitely decreasing to zero (fading), the third is 
the single row. This third sequence satisfies the requirements of both the DWS law and the geometric progression, but does not satisfy the recurrent law. It turns out as an interesting thing, that the DWS law, according to which numerical sequences are formed, actually significantly differs from the recurrent law. It is more significant because it has relation with a larger range of phenomena than a recurrent law. In other words, the DWS law encompasses a recurrent law as an individual case.
3.2. The Nature of the Differences in the Relations Between Two Neighboring Members of Numerical Sequences Formed According to the Both Recurrent Law and the Law of "Doubling with Subtraction"

\subsubsection{The Relation Between Two Neighboring Terms for Numerical Sequences of FB Type, Formed According to the Recurrent Law}

For numerical sequences of FB type the relation between two neighboring terms will be determined as:

$$
\begin{gathered}
\frac{F_{n}}{F_{n-1}}=\frac{F_{n-1}+F_{n-2}}{F_{n-1}}=1+\frac{F_{n-2}}{F_{n-2}+F_{n-3}}=1+\frac{1}{1+\frac{F_{n-3}}{F_{n-2}}}= \\
=1+\frac{1}{1+\frac{F_{n-3}}{F_{n-3}+F_{n-4}}}=1+\frac{1}{1+\frac{1}{1+\frac{F_{n-4}}{F_{n-3}}}}=1+\frac{1}{1+\frac{1}{1+\frac{F_{n-4}}{F_{n-4}+F_{n-5}}}}=1+\frac{1}{1+\frac{1}{1+\frac{1}{1+\frac{F_{n-5}}{F_{n-4}}}}}
\end{gathered}
$$

And so on this computational process can go on forever, forming a complex but interesting mathematical object the value of which tends to $1.618 . .$, to $\Phi$. Perhaps such a mathematical structure can have an importance for mathematics, representing interest as a mathematical object of study.

\subsubsection{Relations Between the Neighboring Two Members of the Sequence, Formed According to the Law of "Doubling with Subtraction"}

Now consider the same situation for the case when the terms of the sequence are formed according to the DWS law. The relations between the neighboring two members in this case will be determined as:

$$
\begin{gathered}
F_{n}=2 F_{n-1}-F_{n-3} ; F_{n-1}=2 F_{n-2}-F_{n-4} \\
\frac{F_{n}}{F_{n-1}}=\frac{2 F_{n-1}-F_{n-3}}{F_{n-1}}=2-\frac{F_{n-3}}{F_{n-1}}= \\
=2-\frac{F_{n-3}}{2 F_{n-2}-F_{n-4}}=2-\frac{F_{n-3}}{2\left(2 F_{n-3}-F_{n-5}\right)-F_{n-4}}= \\
=2-\frac{1}{4-\frac{2 F_{n-5}-F_{n-4}}{F_{n-3}}} \approx \Phi
\end{gathered}
$$

The resulting mathematical construction significantly differs from the structure given above in section 3.2.1. According to the solutions and their results, it can be seen that there are fundamental differences between mathematical expressions that determine the relationship between any two neighboring members of the numerical FB sequence if they are formed according to the recurrent law and the DWS law. In the first case, a certain remainder (surplus) is added to the unit (let it be "a"), in the second case, some other remainder is subtracted from "2" (let it be-"b"). Since it is easy to see that in either case the values of the relations between any two neighboring terms of the sequence are always equal to each other and tends to increase with their ordinal numbers to the number $\Phi$, then the remainders in the mathematical expressions will be: in the first case, $a \simeq 0.618$, in the second, $b \simeq 0.382$. Surprisingly, the remainders $\mathrm{a}$ and $\mathrm{b}$ vary that way that the change in the ordinal numbers of the terms of the sequence does not interrupt absolute equality: $1+a=2-b$. Thereby, a comparative analysis of the DWS law discovered by us with respect to the recurrent law shows that they lead to the same results for sequence of the FB type, although, they are different. Moreover, it should be noted that the DWS law is more important than the recurrent law, because it covers a wider range of phenomena and this law is generalizing while recurrent law is a particular law ensuing from it. As a result, it should be reasonable to say that the DWS law and recurrent law are different and the recurrent law stands as a particular case of the more fundamental and significant DWS law. Behind this mathematical abstract side of these laws real phenomena are hidden which occur in nature and express these laws. Note that the recurrent law has long been represented and still represents, a great mathematical interest as an object of investigation. Many prominent mathematicians have studied it. The DWS law has even more significant mathematical interest is considering what we have noted before. The fact that the DWS law has such a procedure as doubling the previous member of the sequence to determine the subsequent one forming FB sequence seems to be significant for us. This is followed by a possible relation of a given numerical sequence to biophysical phenomena and processes. Therefore, it seemed interesting and important to find a certain model of a branched chain process of a biophysical nature that would be described by a numerical sequence of $\mathrm{FB}$ or other sequences of this type.

\section{Branched Chain Growth Processes of the Rabbit Pairs Number Developing According to the Numerical Fibonacci Sequence, in Accordance with the Law of "Doubling with Subtraction" or with the Main Recurrent Law}

In the nature, a variety of phenomena are observed which patterns of development can correspond to the numerical 
sequences of FB and, in some cases, to the numerical sequences of Lucas, Shannon, triangular numbers and others. The nature of such phenomena can be conditioned and associated with a branched chain patterns or a mechanism embedded in them. Apparently, the prevalence of chemical branched chain reactions, which Semenov N. N. was saying about, can be generally extended to the whole variety of biological and medical processes occurring both naturally and artificially, if we look closely at them, it may turn out that they have a branched chain character. In connection with the stated position that all numerical sequences of the FB type can reflect processes of a different nature that have a branched chain character, it is important to find certain model process that confirms this statement. In this regard, the law of "doubling with subtraction" carries a lot of content. The mechanism of the biological process, which has a branched chain character, is already visible in its form. In any case, the problem of rabbits, which Fibonacci formulated and gave an answer in the form of FB sequence, looks exactly this way. As an active particle in this problem a pair of rabbits can be represented as a whole. A pair of rabbits, like an active particle, leads the chain. It continues the chain and generates new chains producing a branch. Below we will consider the problem of rabbits formulated by Fibonacci which is presented in our interpretation from the point of view of the branched chain character of its process, as well as those surprising consequences to which this problem leads.

\subsection{Biological Model of the Branched Chain Process About Increasing the Number of Rabbit Pairs and Forming a Numerical Fibonacci Sequence, According to the Law of "Doubling with Subtraction"}

As it was already noted, the features of a hypothetical branched chain mechanism that generates the law of development of phenomena in accordance with the numerical FB sequence are viewed in the DWS law. The process of increasing the number of rabbit pairs can relate to such phenomena. Let us note what biophysical meaning can be contained in the expression of this law. In the formula expression of this law, the operation of doubling something previous can be interpreted as a doubling the number of active particles, which are the pairs of rabbits. Previous pairs of rabbits are the number of the pairs which will give offspring. As a result, the number of pairs increases twofold, after all rabbits will give offspring. The action of subtraction can be identified with chain breaks after three acts of chain continuation performed by active particles. With regard to rabbits, it can be considered that all pairs of rabbits on the third occasion will disappear after committing it. Seeing in the resulting ratio a certain biophysical meaning, a mechanism for breeding rabbits was compiled and described on its basis, and an analysis of its functioning with analysis of the obtained results were done. Le us note our other assumptions.

We believe that the newborn pairs of rabbits mature and become capable to reproduce in certain stages and synchronously at the same time as it is necessary for the parent pairs to recover for giving the new offspring. In other words, after the first row of births parenting pairs and newborns simultaneously participate in the next one at the same time. It is quite logical to give this system of births their numbers according to its order: $1 \mathrm{st}, 2 \mathrm{nd}$, 3rd, etc., as we will do later on. Thus, newly born pairs of rabbits enter the next row of births together and simultaneously with the parental pair, which means they become able to bring a new pair in the next row along with the parent couple. We can now describe the branched chain mechanism of breeding rabbits in accordance with such concepts and analyze the results of its functioning. Further, we will make minor clarifications. To select a branched chain mechanism, in accordance with which the process of increasing the number of rabbits during their breeding will be done, it is necessary to determine the conditions. The main concept here, let us emphasize this, is a pair of rabbits, which can be likened to an active particle.

The properties of this active particle (a pair of rabbits) determine the process of increasing the number (of rabbits) according to branched chain mechanisms. Endow it with the following properties. It branches the chain with a branching factor of 2, which means that each pair of rabbits can produce only one pair, which means only reproduce themselves. A pair of rabbits, like an active particle, can create a chain with the length of three, which breaks after it. Such properties of an active particle or a pair of rabbits with connection to the length of a chain in three elements, with its break at the end, are determined by the subtracted second term of the DWS law. This property means that a newborn pair of rabbits which committed three rows of birth disappears from the process. In Table 1, in its first function row, in the order of their numbers, the values of the corresponding terms of the canonical numerical FB sequence are written.

Let us assume that this numerical sequence recorded in the first row reflects the branched chain process of increasing the total number of pairs of rabbits at each stage in accordance with the indicated ordinal numbers. The first and second number are so-called artificially created in the biological process initial conditions for the development of the branched chain process of increasing the total number of rabbit pairs when they are bred. We take any serial number; let it be the fourth number with its value -3 . If the branching factor was equal to two, which would mean doubling the total number of rabbit pairs of the previous step, then in the selected fourth column of the first row there would be a number 4 instead of 3, which means that one pair is missing. Moving on to the fifth column of the first row. According to our thoughts, there should be a number 6 , but there is number 5. Again, one pair is missing. In the sixth column of the first row, in theory, there should be 10 , but we see 8 , this time missing 2 pairs. In the seventh column there should be a 16 , not 13, that is a shortfall of 3 pairs. For the eighth column instead of 21 there should, in theory, be 26 and the shortage is 5 pairs. It is clear that the row of missing pairs (the second functional row of Table 1) also follows the numerical FB sequence and falls behind the main sequence by three 
consecutive numbers.

For greater persuasiveness, we can check this on any member of the main sequence, for example, on the eleventh term of the sequence. Its value is 89 . The value of the previous term (10th) is 55 . Therefore, the value of the term which should have been there is 110 and the number of missing pairs is 21 . This is exactly the value that the 8th member of the sequence has, falling behind 11 th by three numbers (check Table 1). In accordance to our logic this can be understood as the permanent disappearance of those pairs of rabbits that have exhausted their resource for the continuation of chains, limited in length of three links or in the biological sense by three acts of reproduction. A break occurs at the end of any three-link chain, the disappearance of the active particle leading this chain. In Table 1, the third functional row shows the number of newborn rabbits at each step of the process. The filling of this line was done using the logic of branched chain processes with a branching factor -2 . According to this logic, the number of new active particles (pairs of rabbits) appearing at each step is always numerically equal to the number of all active particles (the total number of pairs of rabbits) of the previous step. It is easy to verify that the obtained numerical sequence of the number of rabbit pairs withdrawn from the process is a Fibonacci sequence. This circumstance seems extremely interesting.

Table 1. The branched chain process of incremental growth in the number of rabbit pairs described by the law of "doubling with subtraction".

\begin{tabular}{|c|c|c|c|c|c|c|c|c|c|c|c|c|c|}
\hline & № II/II & 1 & 2 & 3 & 4 & 5 & 6 & 7 & 8 & 9 & 10 & 11 & 12 \\
\hline 1 & Total number of pairs & 1 & 1 & 2 & 3 & 5 & 8 & 13 & 21 & 34 & 55 & 89 & 144 \\
\hline 2 & Tthe number of pairs withdrawn from the process & 0 & 0 & 0 & 1 & 1 & 2 & 3 & 5 & 8 & 13 & 21 & 34 \\
\hline 3 & Newborn pais & 0 & 1 & 1 & 2 & 3 & 5 & 8 & 13 & 21 & 34 & 55 & 89 \\
\hline
\end{tabular}

Table 1 shows that the staged development of the branched chain process described here for all three presented indicators: the total number of pairs, newborn pairs, the number of pairs withdrawn from the process, correspond to the same numerical sequence of FB. The difference between them is that a number of "total numbers" outstrips a number of "newborns" and a number of "withdrawn pairs from the process" by one step and three steps respectively. Thus, the biophysical model of a branched chain process created on the basis of the DWS law reproduces the numerical sequence of FB with respect to the total number of rabbit pairs when they are bred. This model also implies two more important facts regarding the number of newborn couples and pairs withdrawn from the process. The number of both newborn and withdrawn from the process pairs also grows in accordance to the numerical sequence of FB.

\subsection{The Biological Model of the Branched Chain Process of Increasing the Rabbit Pairs Number According to the Recurrent law Which Forms the Numerical Fibonacci Sequence}

Giving the semantic content to the FB sequence using the logic suggested above, according to the new DWS law, allows us to look at the biophysical essence of the main recurrent law in a new way, believing that it reflects its particular branched chain process. Let us set the task to determine what kind of process is it, which recurrent law induces and describes. All the conditions that were used above in the model of branched chain process, which is forming the DWS law, are kept the same. If we examine the essence of FB numbers formation from the same perspective, then we come up with an interesting conclusion that recurrent law describes a branched chain process without chain termination, in other words, with an infinite length. Although, it has one important and necessary condition. This condition lies in the fact that a new emerging particle (a newborn pair of rabbits) becomes able to branch the process only after one step, skipping the next one and after that becoming immortal, leading its chain to infinity. This is the biophysical meaning of the main recurrent law of the FB sequence formation, reflecting the number of newborn rabbits appearing at each step or stage of development of the entire process, with the exception of the first two initial stages. We get a nontrivial answer by speculating using the same logic. So, if the next term for newborn rabbits is the sum of the first two preceding terms, the resulting numerical value for newborns will be the fact that exactly the same number of mature rabbit pairs produced them. Then the total number of newborns and their parents will be exactly twice the number of newborn pairs. Also, there are still immature rabbits in the transition to this stage, that is, newborns in the first preceding stage. So, the total number of rabbits will be determined by the expression: Fn (total) $=2 F n$ (new) + Fn-1 (new), where Fn (new) and Fn-1 (new) are terms of the FB sequence for newborn pairs rabbits, and Fn (total) is the n-th term of the FB sequence for the total number of rabbits. It is not difficult to see that the resulting sequence for the total number of rabbits will also be a FB sequence. In the last expression, instead of the terms from a number of newborn pairs, we can substitute the terms of the total number of pairs and then we will have: Fn (new) $=F n-2$ (total) and Fn-1 (new) $=$ Fn $-3($ total $) ; \mathrm{Fn}($ total $)=2 \mathrm{Fn}-2($ total $)+$ Fn $-3($ total $)=$ Fn -2 $($ total $)+$ Fn-3 (total $)+$ Fn-2 (total) $=$ Fn-1 (total $)+$ Fn-2 (total). This means that the same recurrent law of formation of a numerical sequence is obtained. However, it is necessary to conduct a more detailed analysis of the sequence nature in relation to the total number of pairs and newborn pairs of rabbits at each stage of the whole process. In the same way as it was done above, we will monitor the number of newborn pairs and the total number of pairs and enter the data in Table 2. Start with the initial situation. At the initial moment, there was one mature pair of rabbits, no newborn pairs yet. This moment is reflected in table 2 by putting number 1 in the second row of the first column and number 0 in the first row of the first column. At the second stage a newborn pair 
appeared and it is necessary to reflect this by putting the number 1 in the first row of the second column. The total number of all pairs in this case is two pairs, which is reflected in the cell of the second row and the second column. At the third step, one more newborn pair appears because the newborn pair in the previous step has not reached puberty yet. Therefore, we put number 1 in the first row of the third column. But in the second row and the third column, where total number should be indicated, we put the number 3. At the fourth step, 2 and 5 should be written in the corresponding cells (two of three pairs of the total number created two newborn pairs) and so on.

Table 2. The branched chain process of incremental growth in the number of pairs of rabbits described by recurrent law.

\begin{tabular}{lllllllll}
\hline № & $\mathbf{1}$ & $\mathbf{2}$ & $\mathbf{3}$ & $\mathbf{4}$ & $\mathbf{5}$ & $\mathbf{6}$ & $\mathbf{7}$ & $\mathbf{8}$ \\
\hline Nnewborns & 0 & 1 & 1 & 2 & 3 & 5 & 8 & 13 \\
Ttotal pair number & 1 & 2 & 3 & 5 & 8 & 13 & 21 & 34 \\
\hline
\end{tabular}

Table 2 shows that the phased development of the branched chain process described here by the two presented indexes (the newborn pairs and the total number of pairs), also corresponds to the same numerical FB sequence. The difference between them is that a number of "total numbers" correspond to FB sequence with initial conditions (1.2), and a number of newborn pairs correspond to FB sequence with initial conditions $(0.1)$. We interpret such differences as the delay of a newborn pair number from a total number by two steps. Eventually, we see that it turns out as an amazing case. Two fundamentally different branched chain processes proceed according to completely different laws, which lead to the same result corresponding to the numerical FB sequence. The DWS law generated by a branched chain process with a chain termination and with their three-link length, having a branching factor-2, which is associated with a doubling in the formulation of the law. Other branched chain process proceeds without chain termination with an infinite length, having a branching factor-2. Such a process generates a recurrent law, which characterizes it. These different laws from different processes lead to the same effect - the numerical FB sequence. It is easy to verify using the data of Table 1 that the summation of two FB sequences, reflecting the total number of pairs and newborn pairs leads to a sequence of $1,3,5,8,13$, etc., that is, for $n>3$ the further numerical sequence is a FB sequence, but according to the table 2, the result of this summation is the classical Lucas sequence. Lucas sequence is also obtained from the data of Table 1 when summing up the sequence of the total number of pairs and pairs withdrawn from the process. Indeed, the resulting series $1,2,4,7,11,18,29$, etc., for $n>3$ is the continuation of the Lucas sequence. As a result, we have an amazing case; the Lucas sequence turns out to be obtained from the FB series and it is its derivative. But it is even more surprising that two fundamentally different in nature and content branched chain processes, are described in a similar way by the same DWS law, a which particular version is the recurrent law.

Thus, it is established that the Lucas sequence is determined by summing two Fibonacci sequences shifted by two steps, the first Shannon series, for its part, is determined by summing the two sequences - the Lucas and Fibonacci, also shifted by two steps. The second Shannon sequence is determined by the summation of two, one of which is the first Shannon sequence, and the second one is Lucas sequence, also shifted by two steps. Thus, all sequences are determined through the Fibonacci series. This is an important proposition, indicating that all sequences can reflect branched chain processes according to the type considered for FB. Thus, the biophysical essence of many known numerical sequences characteristics has a similar nature, all of which can reflect the dynamics of the development of branched chain processes with the certain conditions. It should be noted that the DWS law can have other forms besides Fn $=2 F n-1$-Fn-3, for example: $F n=2 F n-1-F n-4 ; F n=2 F n-1-$ Fn-2 and even Fn $=2$ Fn-1 - Fn-1. For each separate form of the DWS law expression there will be a variant of a branched chain process. The form of its expression Fn $=2 \mathrm{Fn}-1-\mathrm{Fn}-3$ describes a branched chain process with a chain termination with a fixed length of three links. If in consideration of the branched chain process of a biological nature there will be other conditions, for example, there will be another situation with the rabbit pair selection, that is, the chain termination conditions will change, then there will be another description of it by numerical sequences. For example, if the length of the chain is not three links (not three acts of continuation of the chain) but two, then the dynamics of such a process, in some cases, will have the form of a numerical sequence of natural numbers. It is easy to verify this by reasoning the same way as it was done before. Let us show what would have happened if, according to the biophysical model of the branched chain process reconstructed by us, pair of rabbits was withdrawn from the reproductive process and disposed only after 2 rows of birth. In this case, the result of determining their number would not be expressed by the numerical FB sequence. It would be expressed by the DWS law, but in a somewhat modified form, namely by: $\mathrm{F}$ (n) = $2 * \mathrm{~F}(\mathrm{n}-1)-\mathrm{F}(\mathrm{n}-2)$. Under the initial conditions (1.1), it would represent a simple single sequence. In the case of the initial conditions $(1,2)$, it would represent a sequence of natural numbers of the form $1,2,3,4,5$, etc. Under the initial conditions $(0,1)$ - this would also be a sequence of natural numbers of the form $0,1,2,3,4,5$, etc. The last circumstance creates some interest. Many people, in accordance with what law the natural number of numbers is formed, will say that it will be expressed as $F(n)=F(n-1)+$ 1 and only few know another kind of the law, namely: $\mathrm{F}(\mathrm{n})=$ $2 * \mathrm{~F}(\mathrm{n}-1)-\mathrm{F}(\mathrm{n}-2)$. And the main thing is that behind this law, which forms a natural sequence of numbers, the reality lies which it was derived of. Thus, the nature of the numerical FB sequence and other considered sequences is that they reflect the dynamics of the development of branched chain processes occurring under strictly defined fixed conditions, a particular example for which is the process of breeding rabbits, described by Fibonacci. 


\section{Conclusion}

The application and use of the FB numbers and the associated with it golden ratio in the biology and medicine has become widespread. Apparently, this is only the beginning, everything will continue to develop. In this regard, the words of Warren Sturgis McCulloch, the famous neurophysiologist, who proposed a formal neuron, which became the prologue to the creation of artificial neural networks, which lies in the basis of artificial intelligence: "I spent two years measuring the ability of a person to bring a controlled oblong subject to the preferred form, because I did not believe that he preferred a golden ratio or that he could recognize it. He prefers and he can!». With respect to the $\mathrm{Fb}$ numbers associated with the golden ratio, the law of their formation, alternative to the recurrent one, is established in the work. This law ("doubling with subtraction", DWS) is valid for all numerical sequences for which the recurrence is also valid. At the same time, there are numerical sequences for which the recurrent law does not work, and the DWS law is valid. This means that the DWS law is more fundamental, and, in fact, is a primary law, and the recurrent law is secondary. Significant differences also exist in the consequences of these two laws. For example, the increments of sequences formed according to the recurrent law and the law of "doubling with subtraction" have fundamental differences in their mathematical expression, although they lead in different ways to the same result, namely, to $\Phi=$ $1,618 \ldots$ with the serial numbers of the terms of the series, which tend to infinity.

In the work, a branched chain biological process that was unique to each one of them was found. In the case of the law of "doubling with subtraction", the process was followed by the termination of chains with characteristic parameters: the chain length is three links, the branching factor is -2 . In the case of a recurrent law, the process was without chain termination, with an infinite length and with a branching factor of -2 , and with some delay limiting the branching. It seems interesting that such different branched chain processes of different character are described by the same Fibonacci sequence. Both these laws are consequences of two fundamentally different branched chain processes. Doubling with subtraction is the consequence of a branched chain process with a chain termination and three-linked length with a branching factor of -2 . The recurrent law is a consequence of a branched chain process without chain termination with an infinite length and a branching factor of 2 , but with the condition that the new emerging active particles become truly active only through a one step. These different consequences from different processes lead to the same result, forming a numerical FB sequence.

As a result, it can be considered justified that the quintessence of many known numerical sequences of the FB type is that they reflect branched chain processes, the spread of which is ubiquitous in nature. Perhaps Fibonacci was the first who drew attention to them.

Interest in different applications of Fibonacci numerical sequences does not decrease. In support of the above, two recent works can be cited $[20,21]$.

\section{References}

[1] Penel N., Kramar A., 2012, What does a modified-Fibonacci dose-escalation actually correspond to?, Medical Research Methodology, 12, 103.

[2] Zelen M., Theory and practive of clinical trials/ 2003, In Holland- Frei Cancer Medicine Ontario BC: Decker.

[3] Barve M. Wang Z., Kumar P. at all/Phase 1 Trial of Bi-shRNA STMN1 BIV in Refractory Cancer/ Molecular Therapy 2015, $23,6,1123-1130$.

[4] Lim H. S., Bae K. S., Jng J. A., at all/ Predicting the efficacy of an oral paclitaxel formulation (DHP107) through modeling and simulation/ Clin. Ther. 2015, 37, (2), 402-417.

[5] Orita M., Ohno K., Niimi T. Two "Golden Ratio" indices in fragment-based drug discovery/Drug Discovery Today, 2009, v. 14 , №5/6, p. 321-328.

[6] Congreve M., Chessari G, Tisi D., Woodhead J./ Resent Developments in Fragment-Based Drug Discovery/Journal of Medicinal Chemistry, 2008, v. 51, №13.

[7] Jarvis G. E., Thompson A. J / A Golden approach to ion channel inhibition, 2013, Trendsin Pharmacological Sciences, v. 34, №9, p. 481-488.

[8] Yamagishi M. E. B, Shimabucuro A. I./. Nucleotide Frequencies in Human Genome and Fibonacci Numbers /J. Mathematical Biology 2008, 70, 3, 643-653.

[9] Forsdyke D. R., Bell S. J. /A discussion of the application of elementary principles to early chemical observations, /Applied Bioinformatics, 2004, 3, 3-8.

[10] Gibson C. M., Gibson W. J., Murphy S. A., Marblt S. J., McCabe C. H., Turakhia M. P., Kirtane A. J., Karha J., Aroesty J. M., Giugliaano R. P., Antman E. M./ Association of the Fibonacci Cascade with the distribution of coronary artery lesions responsible for ST- segment elevation myocardial infarction/ Am. J. Cardiol., 2003, 1, $92(5), 595-597$.

[11] Choo K. W., Quah W. K., Chang G. H., Chan J. Y./ Functional hand proportion is approximated by Fibonacci series/ Folia Morphol (Warsz), 2012, 71 (3), 148-153.

[12] Marinkovic S., Stankovic P., Strbac M., Tomic I., Getkovic M. / Coclea and other spiral forms in nature and art/ Am. J. Otolaryngol. 2012, 33 (1), 80-87.

[13] Visconti G., Visconti E, Bonomo L., Salgarello M./ Concepts in Navel Aesthetic: A Comprehensive Surface Anatomy Analysis/ Aesthetic Plast. Surg., 2015, 39 (1). 43-50.

[14] Yeykin E., Topbas U., Yanik A., Yetkin G./ Does systolic and diastolicblood pressure follow Golden Ratio?/ Int. Cardiol. 2014, 176 (3), 1457-1459.

[15] Stieger S., Swami V. /Time to let go? No automatic aesthetic preference for the golden ratio in art pictures/ Psychology of Aesthetics, Creativity, and the Arts, 2015, v. 9 (1).

[16] Semenov, NN / On Chain Reactions and the Theory of Combustion, Izdatelstvo Znanie 1957, Series VIII, No. 17, Moscow,, p. 1-25; Advances of Chemistry 1957, v. 26, issue 3, p. 273-291). 
[17] Petrenko Yu. M., Vladimirov YA / Determination of the mechanism of action of antioxidants in lipid systems on the parameters of chemiluminescence in the presence of ferrous iron / Biophysics, 1976, vol. 21, №3, p. 424-427.

[18] Titov V. Yu., Petrenko Yu. M., Petrov VA, Vladimirov Yu. A. / Mechanism of oxyhemoglobin oxidation induced by hydrogen peroxide / Byull. Exper. Biol, Medicine, 1991, Vol. 112, No. 7, 46-49.

[19] Shannon A. G./ Generalized Fibonacci Matrices in Medicine/Notes on Number Theoryand Discrete Mathematics $15,2009,1,12-21$.
[20] Iconaru EI, Georgescu L, Ciucurel C./ A mathematical modelling analysis of the response of blood pressure and heart rate to submaximal exercise./ Acta Cardiol. 2018 Jun 18:1-8.

[21] Lee J, Kim ST, Park S, Lee S, Park SH, Park JO, Lim HY, Ahn H, Bok H, Kim KM, Ahn MJ, Kang WK, Park YS./ Phase I Trial of Anti-MET Monoclonal Antibody in METOverexpressed Refractory Cancer./ Clin Colorectal Cancer. 2018 Jun; 17 (2):140-146. 